\title{
Carcinoma of the colon presenting as pulmonary microabscesses and septicaemia due to Clostridium perfringens
}

\author{
LD GRUER, PJ SOUQUET, JM VERGNON, J FLEURETTE, J BRUNE
}

\section{From the Departments of Bacteriology and Respiratory Medicine, Hôpital Cardio-vasculaire et Pneumologique Louis Pradel, Lyon, France}

Occult malignancy may come to light as a result of a pulmonary complication, usually but not always due to metastatic spread. We describe a patient with carcinoma of the colon in whom the diagnosis was suggested by an unusual chest infection and pulmonary emboli.

\section{Case report}

A 63 year old bargee presented in December 1983 with an eight day history of fever, shivering, and a dry cough. He reported that he had had a similar episode five months previously, which had been successfully treated with amoxycillin. A chest radiograph at that time had been normal. Since then he had lost about $9 \mathrm{~kg}$ in weight but had

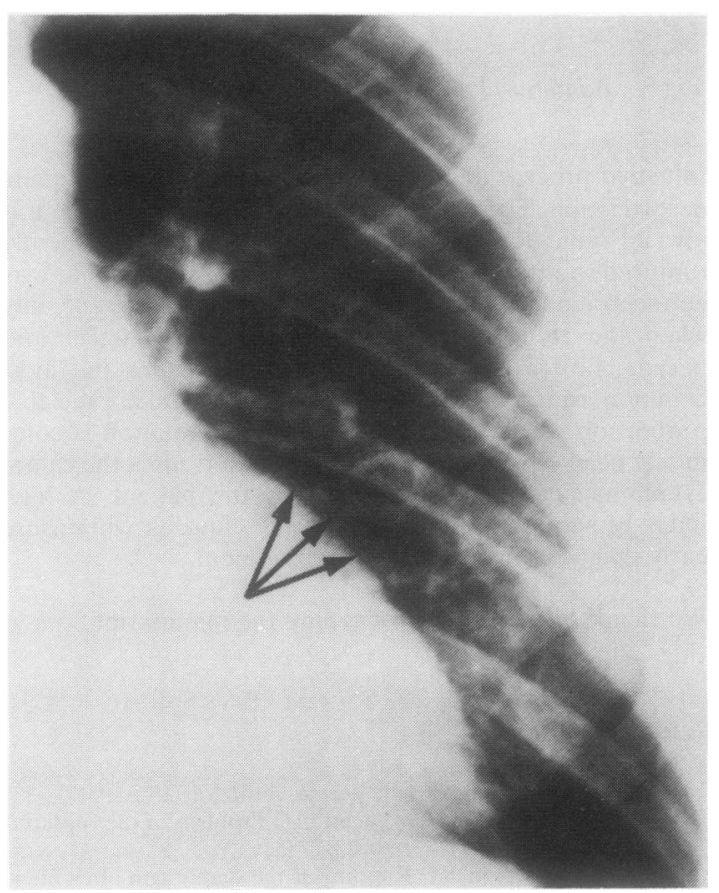

Three small thin walled cavities in the left lung.

Address for reprint requests: Dr LD Gruer, Laboratoire de Bacteriologie Virologie, Faculté de Médecine Alexis Carrel, Rue Guillaume Paradin, 69008 Lyon, France.

Accepted 21 May 1984 had no other symptoms of note. His past medical history was unremarkable and he was a non-smoker. On examination there was evidence of recent weight loss. His temperature was $40.5^{\circ} \mathrm{C}$ and his blood pressure $100 / 60$ (normally $140 / 90) \mathrm{mm} \mathrm{Hg}$, and he had an unproductive cough. There were no other physical signs of note. A chest radiograph with tomograms showed three small, thin walled cavities in the lingula (fig). The white blood cell count was $18 \times 10^{\circ} / 1$, with $80 \%$ polymorphs. Blood cultures grew Clostridium perfringens in both aerobic and anaerobic bottles within 24 hours. At bronchoscopy the bronchial mucosa was diffusely inflamed but no pus was seen. A bronchial aspirate obtained via a sterile catheter yielded a pure growth of $C$ perfringens after 24 hours' anaerobic culture.

He was treated with intravenous benzyl penicillin and within 48 hours was afebrile. Shortly thereafter, however, he developed severe acute bilateral chest pain and became breathless, An electrocardiogram was normal but a ventilation-perfusion lung scan showed perfusion defects in both bases, highly suggestive of pulmonary emboli. Venograms of both legs showed normal appearances. He was given anticoagulant treatment.

It was thought that both the bacteraemia and the pulmonary emboli could be explained by an intestinal lesion. At colonscopy a necrotic polyp was seen in the sigmoid colon and was found to be an adenocarcinoma. At laparotomy three weeks after admission the tumour was found to be associated with a large paracolic abscess. The tumour itself was macroscopically restricted to the intestinal wall. Bacteriological specimens of the abscess were not taken. The abscess was drained and a left hemicolectomy with end to end anastomosis was carried out. The chest radiographic appearances returned to normal, the fever settled and the patient left hospital 15 days after operation.

\section{Discussion}

In a patient with clinical features of septicaemia and radiological signs of acute pulmonary cavitation two principal investigations are required: blood culture and direct sampling of the affected area of lung. In this patient the immediate diagnosis of lung abscess was clear, but unexpectedly he had $C$ perfringens in his blood and his lung. In the absence of necrotic flesh, the most likely source of the $\mathscr{D}$ organism was the large bowel. Suspicion of a pelvic lesion increased when the patient developed bilateral pulmonary emboli without evidence of deep vein thrombosis of the legs. Colonoscopy led quickly to the primary diagnosis and laparotomy revealed the abscess that had undoubtedly caused the bacteraemia and probably also the earlier illness. 
The paracolic abscess was not subjected to microbiological study but we do not think that this detracts from the main message of this report. The patient had anyway received a prolonged course of benzyl penicillin, which might have eliminated clostridia from the region of the tumour. Accordingly, the colonic tumour would remain the probable site of entry of the clostridium whether or not it had been identified at this site.

As the human large bowel invariably contains large numbers of various clostridial species, including $C$ perfringens, ${ }^{\prime}$ inevitably a penetrating bowel lesion will sometimes lead to a $C$ perfringens bacteraemia. Damage to the bowel wall due to recent surgery, carcinoma, radiotherapy, and chemotherapy are the most frequently quoted causes. $^{2-5} C$ perfringens bacteraemia can be rapidly fatal when it occurs in already debilitated patients. In relatively healthy individuals, however, is can be a surprisingly benign and even self limiting condition. ${ }^{2}$ This may be because the anaerobic conditions which clostridia require to express their virulence to the full are found only when local or general tissue oxygenation is severely impaired. Not surprisingly therefore most of the reported instances of $C$ perfringens infection of the lung or pleural space have developed in patients who already had damaged lungs.' In the only recent review of the condition 11 cases were described. ${ }^{6}$ All had major radiological changes, and in all but one it was thought that the organism had been introduced either by inhalation or by transcutaneous contami- nation after thoracocentesis. Only two of the 11 patients died. To our knowledge, however, pulmonary microabscesses caused by $C$ perfringens have not previously been described. Clearly, the condition would go undetected if appropriate samples were not taken and this illustrates the value of direct sampling when localised infection of the lung is suspected.

\section{References}

' Gorbach SL. Other Clostridium species (including gas gangrene). In: Mandell GL, Douglas RG, Bennett JE, eds. Principles and practice of infectious diseases. New York: John Wiley, 1979: 1876-85.

${ }^{2}$ Gorbach SL, Thadepalli H. Isolation of Clostridium in human infections: evaluation of 114 cases. J Infect Dis 1975; 131, suppl: S81-5.

${ }^{3}$ Alpern RJ, Dowell VR. Non histotoxic clostridial bacteremia. Am J Clin Pathol 1971:55:717-22.

${ }^{4}$ Wynne J, Armstrong D. Clostridial septicemia. Cancer 1972; 29:215-21.

${ }^{5}$ Pietrafitta JJ, Deckers PJ. Significance of clostridial bacteraemia. Am J Surg 1982; 143:519-22.

- Bayer AS, Nelson SC, Galpen JE, Chow AW, Guze LB. Necrotising pneumonia and empyema due to Clostridium perfringens. Report of a case and review of the literature. Am J Med 1975; 59:851-6. 